ARTICLE

https://doi.org/10.1038/s41467-020-20252-7

\title{
Strain-stabilized superconductivity
}

\author{
J. P. Ruf(1) ${ }^{1 凶}$, H. Paik ${ }^{2,3}$, N. J. Schreiber ${ }^{3}$, H. P. Nair ${ }^{3}$, L. Miao ${ }^{1}$, J. K. Kawasaki (1) 1,4 , J. N. Nelson (1) 1, B. D. Faeth ${ }^{1,2}$, \\ Y. Lee ${ }^{1}$, B. H. Goodge (1) 5,6 , B. Pamuk ${ }^{5}$, C. J. Fennie (1) ${ }^{5}$, L. F. Kourkoutis (1) ${ }^{5,6}$, D. G. Schlom (1) ${ }^{3,6,7}$ \& \\ K. M. Shen (1) ${ }^{1,6 \times}$
}

Superconductivity is among the most fascinating and well-studied quantum states of matter. Despite over 100 years of research, a detailed understanding of how features of the normalstate electronic structure determine superconducting properties has remained elusive. For instance, the ability to deterministically enhance the superconducting transition temperature by design, rather than by serendipity, has been a long sought-after goal in condensed matter physics and materials science, but achieving this objective may require new tools, techniques and approaches. Here, we report the transmutation of a normal metal into a superconductor through the application of epitaxial strain. We demonstrate that synthesizing $\mathrm{RuO}_{2}$ thin films on (110)-oriented $\mathrm{TiO}_{2}$ substrates enhances the density of states near the Fermi level, which stabilizes superconductivity under strain, and suggests that a promising strategy to create new transition-metal superconductors is to apply judiciously chosen anisotropic strains that redistribute carriers within the low-energy manifold of $d$ orbitals.

\footnotetext{
${ }^{1}$ Department of Physics, Laboratory of Atomic and Solid State Physics, Cornell University, Ithaca, NY 14853, USA. ${ }^{2}$ Platform for the Accelerated Realization, Analysis, and Discovery of Interface Materials, Cornell University, Ithaca, NY 14853, USA. ${ }^{3}$ Department of Materials Science and Engineering, Cornell University, Ithaca, NY 14853, USA. ${ }^{4}$ Department of Materials Science and Engineering, University of Wisconsin, Madison, WI 53706, USA. ${ }^{5}$ School of Applied and Engineering Physics, Cornell University, Ithaca, NY 14853, USA. ${ }^{6}$ Kavli Institute at Cornell for Nanoscale Science, Ithaca, NY 14853, USA.

7 Leibniz-Institut für Kristallzüchtung, Max-Born-Str. 2, Berlin 12489, Germany. ${ }^{凶}$ email: jpr239@cornell.edu; kmshen@cornell.edu
} 
n typical weak-coupling theories of superconductivity, the effective attraction $V$ between electrons is mediated by the exchange of bosons having a characteristic energy scale $\omega_{\mathrm{B}}$, and superconductivity condenses below a transition temperature $T_{c}$ parameterized as ${ }^{1}$ :

$$
T_{c} \sim \omega_{\mathrm{B}} \exp \left(-\frac{1}{N\left(E_{F}\right) V}\right)=\omega_{\mathrm{B}} \exp \left(-\frac{1+\lambda}{\lambda-\mu^{*}}\right),
$$

where $N\left(E_{F}\right)$ is the density of states (DOS) near the Fermi level, $\lambda$ is the electron-boson coupling strength, and $\mu^{*}$ is the Coulomb pseudopotential that describes the residual Coulomb repulsion between quasiparticles ${ }^{2}$. For simplicity, we assume that all of the non-isotropic q- and $\mathbf{k}$-dependencies that appear in a more realistic formulation of Cooper pairing have been averaged away. Note that within the range of validity of Eq. (1) - viz., $1 \gg \lambda>\mu^{*}$ -increasing $\lambda$ (increasing $\mu^{*}$ ) generally enhances (suppresses) $T_{\mathcal{c}}$, respectively, assuming that superconductivity remains the dominant instability.

Experimental methods that boost $T_{c}$ are highly desired from a practical perspective. Furthermore, by analyzing how these available knobs couple to the normal-state properties on the right side of Eq. (1), one can envisage engineering the electronic structure and electron-boson coupling to optimize $T_{c}$. For example, increasing $N\left(E_{F}\right)$ is a frequently suggested route towards realizing higher $T_{c}$, but how to achieve this for specific materials often remains unclear.

Historically, chemical doping and hydrostatic pressure have been the most common knobs used to manipulate superconductivity. Unfortunately, doping has the complication of explicitly introducing substitutional disorder, whereas pressure studies are incompatible with most probes of electronic structure. Moreover, because large pressures are usually required to appreciably increase $T_{c}^{3}$, pressure-enhanced superconductivity exists transiently-oftentimes in different structural polymorphs than at ambient conditions-rendering it inaccessible for applications.

An alternative strategy for controlling superconductivity is epitaxial strain engineering. This approach is static, disorder-free, allows for the use of sophisticated experimental probes ${ }^{4}$, and enables integration with other materials in novel artificial interfaces and device structures ${ }^{5,6}$. To date, epitaxial strain has only been used to modulate $T_{c}$ in known superconductors ${ }^{7-12}$. In this article, we describe the creation of a new superconductor through epitaxial strain, starting from a compound, $\mathrm{RuO}_{2}$, previously not known to be superconducting. By comparing the results of angleresolved photoemission spectroscopy (ARPES) experiments with density functional theory (DFT) calculations, we show that splittings between the effective low-energy $d$ orbital degrees of freedom in $\mathrm{RuO}_{2}$ respond sensitively to appropriate modes of strain, and we discuss how this approach may open the door to strain tuning of superconductivity in other materials.

\section{Results}

Electrical and structural characterization of $\mathrm{RuO}_{2}$ thin films. Bulk $\mathrm{RuO}_{2}$ crystallizes in the ideal tetragonal rutile structure (space group $\# 136, P 4_{2} / \mathrm{mnm}$ ) with lattice constants at $295 \mathrm{~K}$ of $(a=4.492 \AA, c=3.106 \AA)^{13}$. $\mathrm{RuO}_{2}$ thin films in distinct epitaxial strain states were synthesized using oxide molecular-beam epitaxy (MBE) by employing different orientations of isostructural $\mathrm{TiO}_{2}$ substrates, $(a=4.594 \AA, c=2.959 \AA)^{14}$. As shown in Fig. 1a, $b$, the surfaces of (101)-oriented substrates are spanned by the $[\overline{101}]$ and [010] lattice vectors of $\mathrm{TiO}_{2}$, which ideally impart in-plane tensile strains on $\mathrm{RuO}_{2}$ (at $295 \mathrm{~K}$ ) of $+0.04 \%$ and $+2.3 \%$, respectively. $\mathrm{On} \mathrm{TiO}_{2}(110)$, the lattice mismatches with $\mathrm{RuO}_{2}$ are larger: $-4.7 \%$ along [001] and $+2.3 \%$ along [110].
Figure 1c shows electrical resistivity $\rho(T)$ measurements for $\mathrm{RuO}_{2}$ films, along with results for bulk $\mathrm{RuO}_{2}$ single crystals from Ref. ${ }^{15}$. To compare with bulk, for the thin-film samples we plot the geometric mean of the components of $\rho$ along the two inplane directions; the intrinsic resistive anisotropy is known to be small $^{16}$, consistent with our findings (Supplementary Note 1 and Supplementary Fig. 1). $\rho(T)$ data for the lightly strained $\mathrm{RuO}_{2} /$ $\mathrm{TiO}_{2}(101)$ sample-henceforth referred to as $\mathrm{RuO}_{2}(101)$-are nearly indistinguishable from bulk, exhibiting metallic behavior with a low residual resistivity $\rho(0.4 \mathrm{~K})<2 \mu \Omega$-cm. In contrast, a clear superconducting transition is observed for the more heavily strained $\mathrm{RuO}_{2} / \mathrm{TiO}_{2}(110)$ sample-referred to as $\mathrm{RuO}_{2}(110)$-at $T_{c}=2.0 \pm 0.1 \mathrm{~K}$.

Magnetoresistance measurements (Fig. 1e, f) with $H_{\perp}$ applied along [110] (the out-of-plane direction) show a monotonic suppression of $T_{c}$ with increasing fields and an extrapolated value of $H_{c \perp}(T \rightarrow 0 \mathrm{~K})=13.3 \pm 1.5 \mathrm{kOe}$, corresponding to an average in-plane superconducting coherence length of $\xi(T \rightarrow 0 \mathrm{~K})=15.8 \pm 0.9 \mathrm{~nm}$ (Supplementary Note 2 and Supplementary Fig. 2). In Fig. 1d, we show a $V(I)$ curve measured on a lithographically patterned resistivity bridge at $T / T_{c}=0.3$, from which we extract a critical current density $J_{c}=(9.5 \pm 1.2) \times 10^{4}$ $\mathrm{A} / \mathrm{cm}^{2}$. This large value of $J_{c}$ (over one order of magnitude larger than values reported on typical elemental superconductors with comparable $T_{c} s$ ) indicates that the superconductivity in $\mathrm{RuO}_{2}(110)$ does not arise from a filamentary network, structural defects, minority phases, or from the substrate-film interface, which would all yield much smaller values of $J_{c}$.

In order to disentangle the effects of strain from other possible sources of superconductivity, we compare $\mathrm{RuO}_{2}$ films as functions of strain and film thickness, $t$. In Fig. $2 a$, we plot $x^{-}$ ray diffraction (XRD) data from similar-thickness films of $\mathrm{RuO}_{2}(101)$ and $\mathrm{RuO}_{2}(110)$, showing that the bulk-averaged crystal structures of the films are strained as expected along the out-of-plane direction based on their net in-plane lattice mismatches with $\mathrm{TiO}_{2}$. The primary 101 and 202 film peaks of $\mathrm{RuO}_{2}(101)$ are shifted to larger angles than bulk $\mathrm{RuO}_{2}$, corresponding to a $1.1 \%$ compression of $d_{101}$, while NelsonRiley analysis of the primary 110, 220, and 330 (see, e.g., Supplementary Fig. 4) peak positions for $\mathrm{RuO}_{2}(110)$ evidence a $2.0 \%$ expansion of $d_{110}$ relative to bulk. In Fig. $2 b, c$, we plot resistivity data showing that reducing $t$ in $\mathrm{RuO}_{2}(110)$ decreases $T_{c}$, as is commonly observed in numerous families of thin-film superconductors ${ }^{17,18}$, with $T_{c}$ dropping below our experimental threshold $(0.4 \mathrm{~K})$ between $t=11.5$ and $5.8 \mathrm{~nm}$. This suppression of $T_{c}$ with thickness indicates superconductivity is not confined near the substrate-film interface, so possible interfacial modifications of the crystal structure ${ }^{19}$, carrier density ${ }^{20}$, substrate-film mode coupling ${ }^{21}$, and non-stoichiometry in the films or substrates $22-24$ can all be eliminated as potential causes of superconductivity. These conclusions are also supported by the facts that superconductivity is not observed in $\mathrm{RuO}_{2}(101)$ films, nor in bare $\mathrm{TiO}_{2}$ substrates treated in an identical fashion to the $\mathrm{RuO}_{2}$ films. Finally, in Fig. 2d we include a scanning transmission electron microscopy (STEM) image of a superconducting $\mathrm{RuO}_{2}(110)$ sample, which confirms uniform growth of the film over lateral length scales exceeding those expected to be relevant for superconductivity (e.g., $\xi$ ), and shows a chemically abrupt interface between $\mathrm{RuO}_{2}$ and $\mathrm{TiO}_{2}$ (Supplementary Fig. 5), with no evidence of minority phases.

We believe the thickness dependence of $T_{c}$ results primarily from the competition between: (i) an intrinsic strain-induced enhancement of $T_{c}$ that should be maximized for thinner, commensurately strained $\mathrm{RuO}_{2}(110)$ films, versus (ii) disorderinduced suppressions of $T_{c}$ that become amplified in the ultrathin limit (see, e.g., $\rho_{0}$ versus $t$ in Fig. 2c). While the thinnest films 


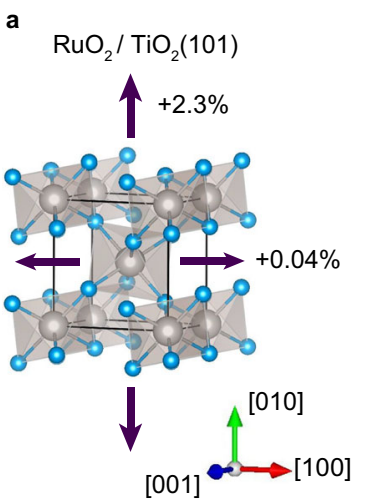

b

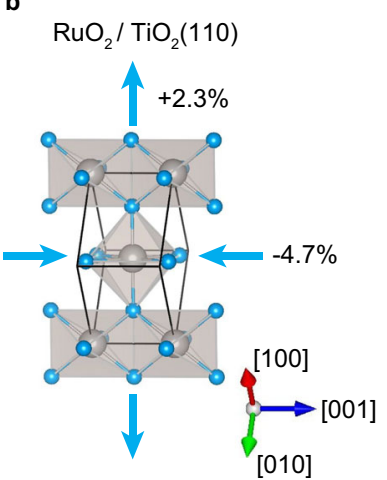

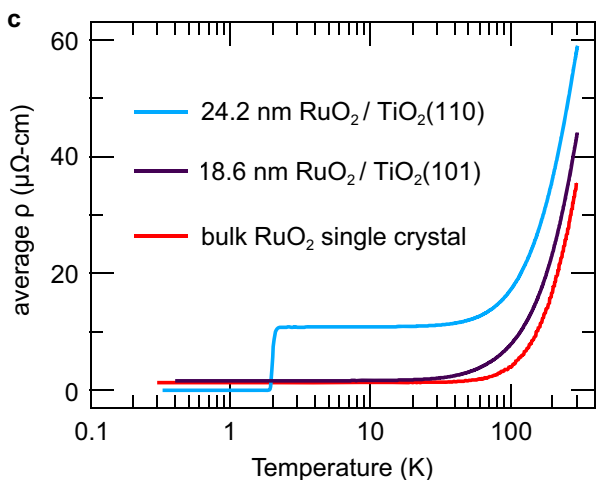
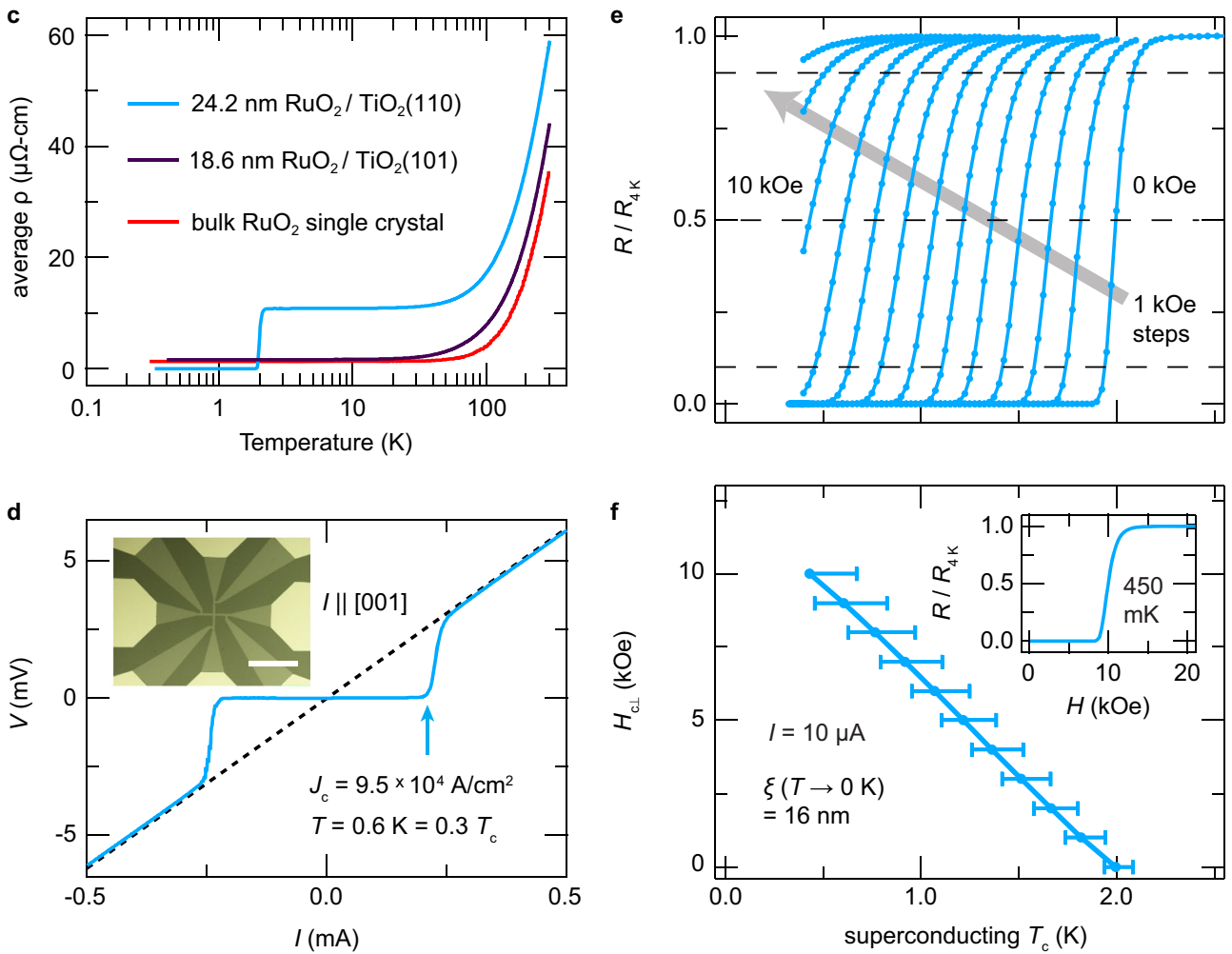

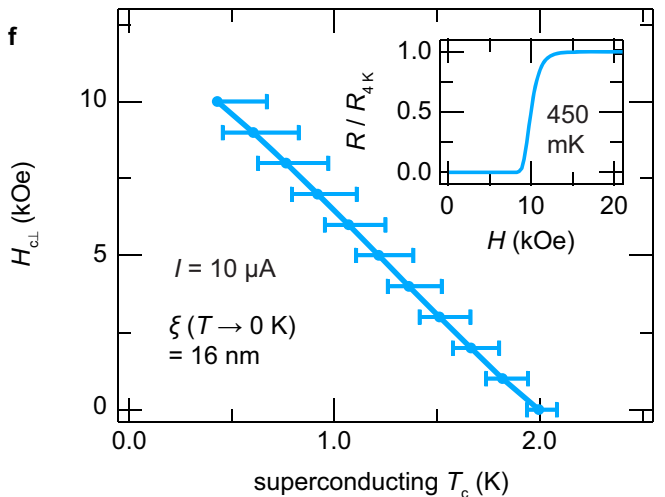

Fig. 1 Electrical transport behavior of bulk $\mathbf{R u O}_{\mathbf{2}}$ single crystals and epitaxially strained $\mathbf{R u O}_{\mathbf{2}}$ thin films. $\mathbf{a}$, $\mathbf{b}$ Schematic diagrams of the crystal structures and in-plane lattice mismatches with $\mathrm{TiO}_{2}$ substrates of $\mathrm{RuO}_{2}$ thin films synthesized in (101)- and (110)-orientations. Gray and blue spheres represent $\mathrm{Ru}$ and $\mathrm{O}$ atoms, respectively. c Average resistivity versus temperature curves for $24.2 \mathrm{~nm}$ thick $\mathrm{RuO}_{2}(110)$ and $18.6 \mathrm{~nm}$ thick RuO ${ }_{2}(101)$ films, compared to results for bulk $\mathrm{RuO}_{2}$ single crystals from Ref. ${ }^{15}$. For clarity the bulk $\mathrm{RuO}_{2}$ data have been rigidly shifted upward by $1 \mu \Omega$-cm $\left(\rho_{0} \approx 0.3 \mu \Omega\right.$-cm). d $V(I)$ curve measured at $0.6 \mathrm{~K}$ on a $10 \mu \mathrm{m}$-wide resistivity bridge lithographically patterned on the $\mathrm{RuO}_{2}(110)$ sample from (c) (as shown in the inset: scale bar $=200 \mu \mathrm{m}$ ), which has the direction of current flow parallel to [001] $]_{\text {rutile. }}$ Similarly large critical current densities $J_{c}$ are obtained with $/ \|[1 \overline{0} 0]$ (Supplementary Note 1 and Supplementary Fig. 1). e, $\mathbf{f}$ Upper critical magnetic fields $H_{c \perp}$ versus superconducting $T_{c} s$ extracted from magnetoresistance measurements for the $\mathrm{RuO}_{2}(110)$ sample in (c) along with a characteristic $R(H)$ sweep acquired at $0.45 \mathrm{~K}$ (inset in (f)). Superconducting $T_{c} \mathrm{~s}$ are taken as the temperatures at which the resistance crosses $50 \%$ of its residual normal-state value $R_{4}$ k; error bars on these $T_{c} s$ indicate where $R$ crosses the $90 \%$ and $10 \%$ thresholds of $R_{4} \mathrm{~K}$, respectively (cf. the horizontal dashed lines in (e)).

experience the largest substrate-imposed strains, stronger disorder scattering (likely from interfacial defects) reduces $T_{c}$ below our detection threshold. Films of intermediate thickness $(t \approx$ $10-30 \mathrm{~nm}$ ) have lower residual resistivities and higher $T_{c} \mathrm{~s}$, but do exhibit signatures of partial strain relaxation. Nevertheless, a detailed analysis of misfit dislocations by STEM and XRD reciprocal-space mapping (Supplementary Notes 3, 4 and Supplementary Figs. 8-10) indicates that these films are largely structurally homogeneous and, on average, much closer to commensurately strained than fully relaxed. Finally, in much thicker samples (e.g., $t=48 \mathrm{~nm}$ ) where a more significant volume fraction of the film should be relaxed, the strain is further released by oriented micro-cracks that make such samples spatially inhomogeneous and cause severely anisotropic distributions of current flow, preventing reliable resistivity measurements (Supplementary Fig. 11).

DFT calculations and ARPES measurements. Having established the strain-induced nature of the superconductivity in $\mathrm{RuO}_{2}(110)$, we now explore its underlying origin using a combination of DFT and ARPES. In Fig. 3a, we present the electronic structure of commensurately strained $\mathrm{RuO}_{2}(110)$ calculated by DFT $+U(U=2 \mathrm{eV})$, following the methods of Berlijn et al. ${ }^{13}$. Despite being constructed of $\mathrm{RuO}_{6}$ octahedra having the same $4 d^{4}$ electronic configuration as in $(\mathrm{Ca}, \mathrm{Sr}, \mathrm{Ba}) \mathrm{RuO}_{3}$, the electronic structure of $\mathrm{RuO}_{2}$ is markedly different from that of perovskite- based ruthenates. These distinctions arise from a sizable ligandfield splitting of the $t_{2 g}$ orbitals, such that the most natural description of the low-energy electronic structure is in terms of states derived from two distinct types of orbitals: $d_{\|}$and $\left(d_{\mathrm{xz}}, d_{\mathrm{yz}}\right)$, as illustrated by plots of Wannier functions in Fig. $3 b^{25,26}$. Viewed in the band basis in Fig. 3a, the differentiation in $\mathbf{k}$-space between these orbitals becomes apparent: the near- $E_{F} d_{\|}$states (yellow-orange) form mostly flat bands concentrated around the $k_{001}=\pi / c$ (i.e., Z-R-A) plane, whereas the $\left(d_{\mathrm{xz}}, d_{\mathrm{yz}}\right)$ states (purple) form more isotropically dispersing bands distributed uniformly throughout the Brillouin zone.

In many other $d^{4}$ ruthenates (such as $\mathrm{Sr}_{2} \mathrm{RuO}_{4}$ and $\mathrm{Ca}_{2} \mathrm{RuO}_{4}$ ), static mean-field electronic structure calculations (such as DFT $+U$ ) often predict quantitatively incorrect effective masses ${ }^{27-31}$-and sometimes even qualitatively incorrect ground states $^{32}$-because these approaches neglect local (atomic-like) dynamical spin-orbital correlations (driven by Hund's rules) that strongly renormalize the low-energy quasiparticle excitations. Therefore, it is imperative to compare DFT calculations for $\mathrm{RuO}_{2}$ with experimental data, to establish the reliability of any theoretically predicted dependence of the electronic structure on strain. The left half of Fig. $3 \mathrm{~d}$ shows the Fermi surface of $\mathrm{RuO}_{2}(110)$ measured with $\mathrm{He}-\mathrm{I} \alpha(21.2 \mathrm{eV})$ photons at $17 \mathrm{~K}$, which agrees well with a non-magnetic DFT $+U$ simulation of the Fermi surface at a reduced out-of-plane momentum of $k_{110}=-0.2 \pm 0.2 \pi / d_{110}$ (right half of Fig. $3 \mathrm{~d}$ ). In Fig. $3 \mathrm{e}, \mathrm{f}$, we 

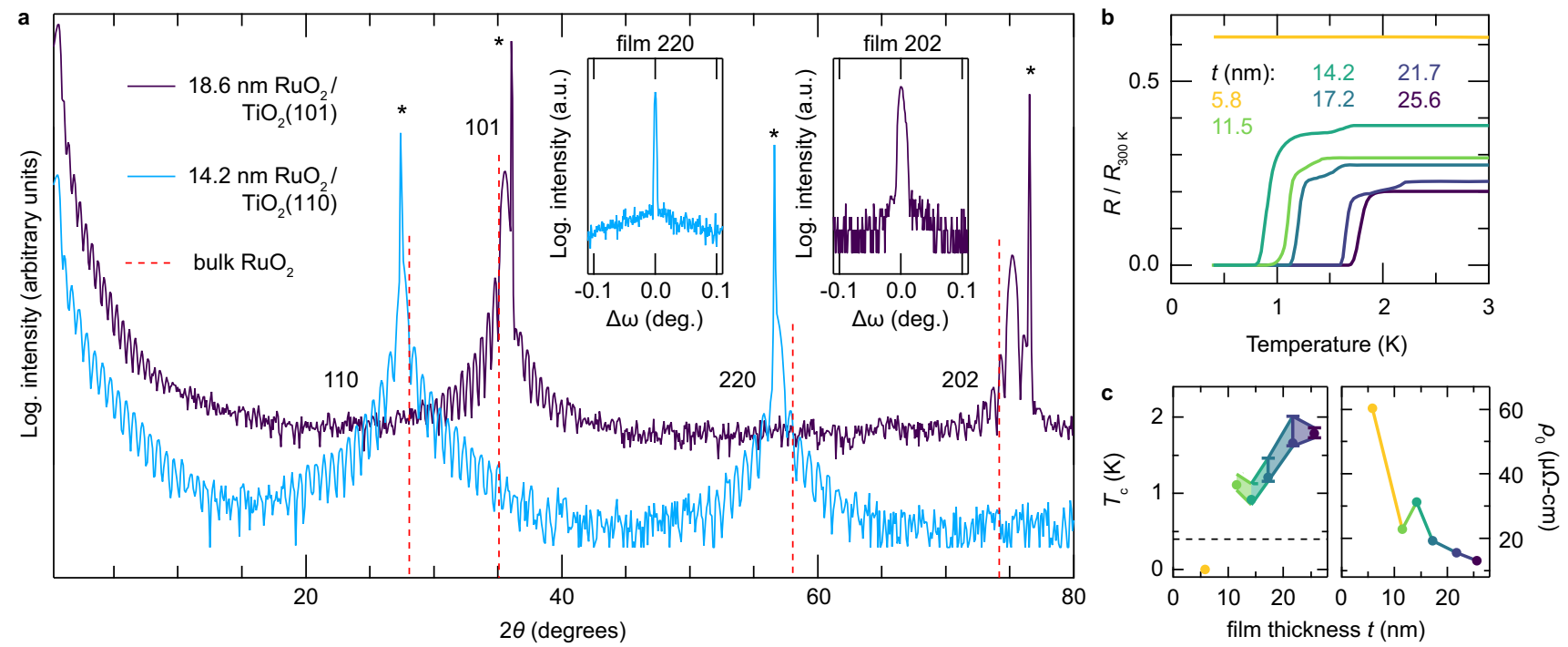

d

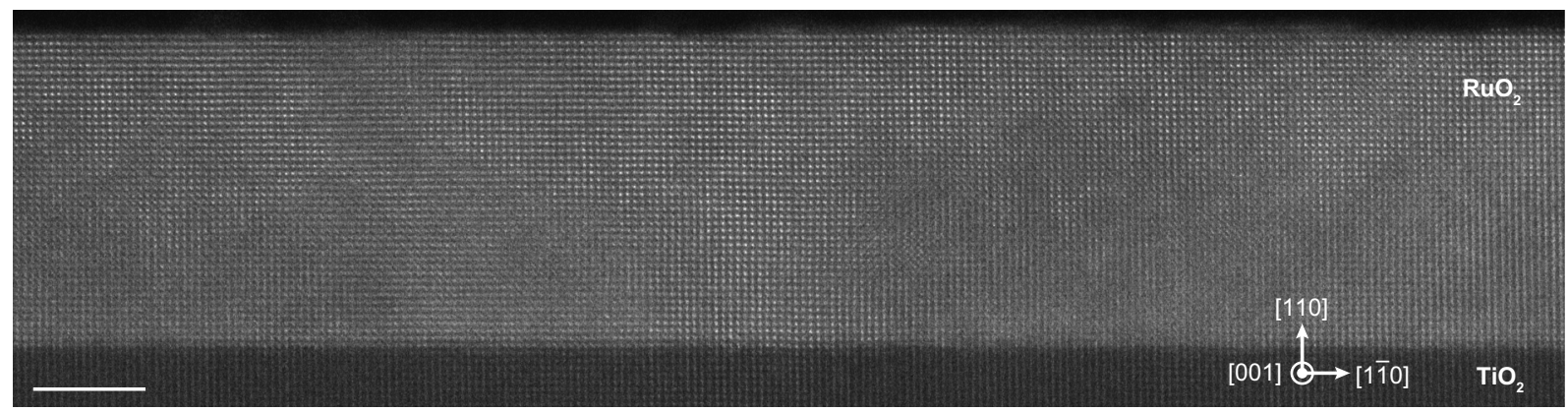

Fig. 2 Structural characterization of epitaxially strained $\mathrm{RuO}_{2}$ thin films, and film-thickness-dependent superconductivity for $\mathrm{RuO}_{2}(110)$. a $\mathrm{XRD}$ data acquired with $\mathrm{Cu}-\mathrm{K} \alpha$ radiation along the specular crystal truncation rods for $18.6 \mathrm{~nm}$ thick $\mathrm{RuO}_{2}(101)$ and $14.2 \mathrm{~nm}$ thick RuO $\mathrm{O}_{2}(110)$ films. Bragg peaks arising from the $\mathrm{TiO}_{2}$ substrates are marked with asterisks, and the peak positions that would be expected for unstrained bulk RuO $\mathrm{O}_{2}$ are indicated by dashed red lines $^{13}$. Insets display rocking curves with FWHMs $<0.01^{\circ}$ acquired at the $2 \theta$ values corresponding to the primary 220 and 202 film peaks. Here $q_{\|}$is aligned with $\mathrm{TiO}_{2}[1 \overline{1} 0]$ for the (110)-oriented sample, and with $\mathrm{TiO}_{2}[1 \overline{101}]$ for the (101)-oriented sample. b Resistance versus temperature curves for $\mathrm{RuO}_{2}(110)$ samples with different film thicknesses $t$, normalized to their values at $300 \mathrm{~K}$. c Superconducting $T_{c} \mathrm{~s}$ and residual resistivities $\rho_{0}$ plotted versus film thickness for the $\mathrm{RuO}_{2}(110)$ samples from (b). Error bars on $T_{c} \mathrm{~s}$ have the same meaning as in Fig. 1. The horizontal dashed line represents the base temperature attainable in our refrigerator, $0.4 \mathrm{~K}$. d STEM image of the same $14.2 \mathrm{~nm}$ thick $\mathrm{RuO}_{2}(110)$ sample from (a-c) (scale bar $=5 \mathrm{~nm}$ ). More comprehensive structural and electrical characterization of the samples shown here are included in Supplementary Notes 3, 4 and Supplementary Figs. 3-10.

plot energy versus momentum spectra acquired along the white dashed lines in Fig. 3d: in Fig. 3e, the spectrum is dominated by the flat $d_{\|}$bands centered around a binding energy of $300 \mathrm{meV}$, whereas in Fig. 3f the $\left(d_{\mathrm{xz}}, d_{\mathrm{yz}}\right)$-derived bands are steeply dispersing and can be tracked down to several hundred meV below $E_{F}$, both of which are well reproduced by DFT $+U$ calculations. The reasonable agreement between the experimentally measured and DFT band velocities is consistent with recent ARPES studies of Ir-doped $\mathrm{RuO}_{2}$ single crystals ${ }^{33}$ and with earlier specific heat measurements of the Sommerfeld coefficient in bulk $\mathrm{RuO}_{2}$, which suggested a modest momentum-averaged quasiparticle mass renormalization of $\gamma_{\exp }=1.45 \gamma_{\mathrm{DFT}}{ }^{34,35}$. The fact that the true electronic structure of $\mathrm{RuO}_{2}$ can be well accounted for by DFT $+U$ allows us to utilize such calculations to understand how epitaxial strains can be employed to engineer features of the electronic structure to enhance the instability towards superconductivity.

Evolution of electronic structure under strain. In Fig. 4a, we show the strain dependence of the DFT-computed band structure and DOS for $\mathrm{RuO}_{2}(110), \mathrm{RuO}_{2}(101)$, and bulk $\mathrm{RuO}_{2}$. While the results for $\mathrm{RuO}_{2}(101)$ are almost identical to bulk, the results for $\mathrm{RuO}_{2}(110)$ exhibit significant differences: the large $d_{\| \text {-derived }}$ peak in the DOS (centered around a binding energy of $800 \mathrm{meV}$ for bulk) is split into multiple peaks for $\mathrm{RuO}_{2}(110)$, several of which are shifted closer to the Fermi level, thereby increasing $N\left(E_{F}\right)$. In our studies, we found that this strain-dependent trend was robust against details of the DFT calculations, such as whether $U$ was finite (Supplementary Note 5 and Supplementary Fig. 12). In order to determine whether this strain dependence of $N\left(E_{F}\right)$ is realized in experiment, we compared the electronic structure of a thin $(7 \mathrm{~nm})$ highly strained $\mathrm{RuO}_{2}(110)$ film with a much thicker (48 nm) partially strain-relaxed $\mathrm{RuO}_{2}(110)$ film. The surface lattice constants of the $48 \mathrm{~nm}$ thick film were closer to bulk $\mathrm{RuO}_{2}$ than the $7 \mathrm{~nm}$ thick film (Supplementary Note 7 and Supplementary Fig. 14), so we expect that the surface electronic structure probed by ARPES of the thicker film to be more representative of bulk $\mathrm{RuO}_{2}$. Comparisons between the $\mathrm{RuO}_{2}(110)$ and $\mathrm{RuO}_{2}(101)$ surfaces are less straightforward, since different parts of the three-dimensional Brillouin zone are sampled by ARPES (Supplementary Note 8 and Supplementary Fig. 15). Figure $4 \mathrm{~b}$ shows $E(k)$ spectra side by side for the $7 \mathrm{~nm}$ (left) and $48 \mathrm{~nm}$ (right) films of $\mathrm{RuO}_{2}(110)$ along the same cut through k-space from Fig. 3e where the photoemission intensity is dominated by $d_{\|}$initial states. The higher levels of strain present at the film surface for the $7 \mathrm{~nm}$ thick sample cause a substantial shift of the flat bands towards $E_{F}$ by $120 \pm 20 \mathrm{meV}$ relative to the more strain-relaxed $48 \mathrm{~nm}$ thick sample. Integrating the 

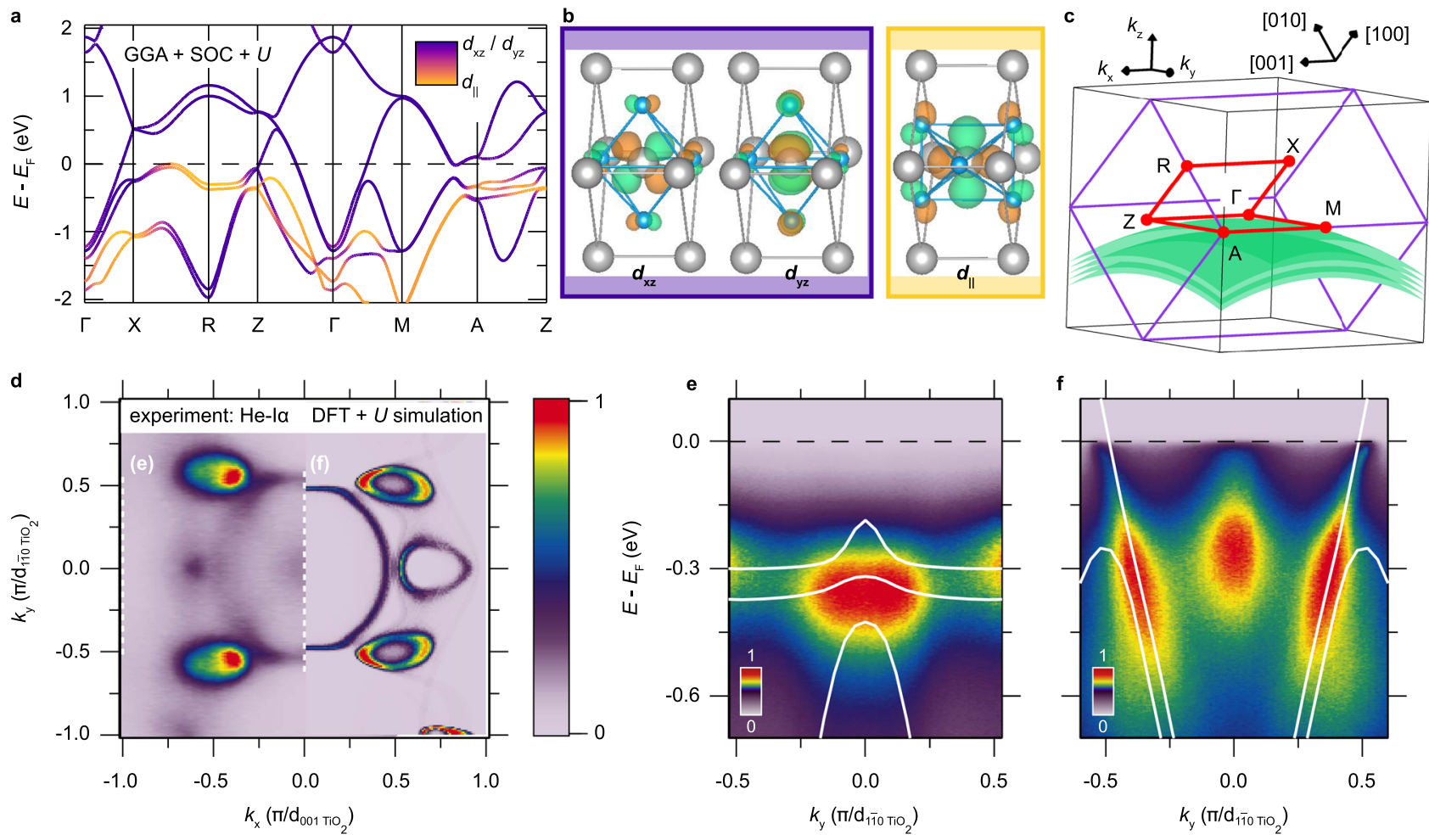

Fig. 3 Electronic structure of $\mathbf{R u O}_{2}$. a Non-magnetic band structure of $\mathrm{RuO}_{2}(110)$ according to $\mathrm{DFT}$, calculated within the generalized gradient approximation (GGA) including spin-orbit coupling (SOC) and a static $+U=2 \mathrm{eV}$ correction on the Ru sites. The color scale indicates the magnitudes of projections of the Kohn-Sham eigenstates at each $\mathbf{k}$ onto Ru-centered Wannier functions with $d_{\|}$and $\left(d_{\mathrm{xz}}, d_{\mathrm{yz}}\right)$ orbital characters, which are constructed from the manifold of self-consistent eigenstates spanning $E_{F}$ and are plotted in drawings of the crystal structure in $(\mathbf{b})$. Ru $(O)$ atoms are colored gray (blue), as in Fig. 1a, b. Green and orange surfaces in (b) represent isosurfaces of the Wannier functions that have equal absolute magnitudes, but opposite (i.e., positive and negative) signs, respectively. c Brillouin zone schematic defining the coordinate system utilized for describing ARPES measurements of

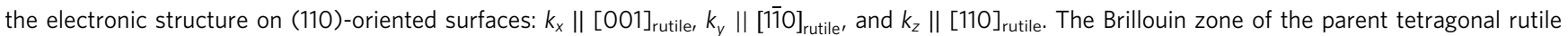
structure is outlined in purple, the high-symmetry contour for the spaghetti plot from (a) is colored red, and the region probed on (110)-oriented surfaces with $\mathrm{He}-\mathrm{l} \alpha$ photons $(21.2 \mathrm{eV}$ ) is shaded green (Supplementary Note 6 and Supplementary Fig. 13). d Slice through the Fermi surface experimentally measured for a $7 \mathrm{~nm}$ thick $\mathrm{RuO}_{2}(110)$ film (left), compared to the Fermi surface from DFT $+U$ simulations (right) projected onto the region of the Brillouin zone colored green in $(\mathbf{c}) . E(k)$ spectra acquired along the one-dimensional cuts indicated by dashed white lines in (d) show: e flat bands with $d_{\|}$orbital character and $\mathbf{f}$ more dispersive bands with $\left(d_{\mathrm{xz}}, d_{\mathrm{yz}}\right)$ character, both consistent with DFT $+U$ expectations (solid white lines). The intensities of the experimental data shown in (d-f) and of the DFT simulations shown in (d) are plotted in arbitrary units where we define 0 (1) to be the minimum (maximum) value, respectively, of the given data set. Only relative changes in intensity within a given panel (as visualized by the false color scales) are meaningful.

ARPES data over the full measured region of $\mathbf{k}$-space for both samples gives the average energy distribution curves plotted in Fig. $4 \mathrm{c}$, which show that spectral weight near $E_{F}$ is enhanced as the $d_{\| \mid}$states move towards $E_{F}$, in qualitative agreement with the trend predicted by DFT. Our results indicate that the primary electronic effect of the epitaxial strains in $\mathrm{RuO}_{2}(110)$ is to alter the relative occupancies of the $d_{\|}$and $\left(d_{\mathrm{xz}}, d_{\mathrm{yz}}\right)$ orbitals as compared with bulk, and to push a large number of states with $d_{\|}$ character closer to $E_{F}$, which enhances $N\left(E_{F}\right)$ and likely $T_{c}$.

\section{Discussion}

Observations of Fermi-liquid-like quasiparticles near $E_{F} 34,36-38$ that scatter at higher energies primarily via their interaction with phonons ${ }^{16,35}$, along with the fact that superconductivity in $\mathrm{RuO}_{2}(110)$ persists in the dirty limit (Supplementary Note 4 and Supplementary Fig. 9), are both consistent with conventional Cooper pairing, suggesting that calculations assuming an electron-phonon mechanism may be enlightening. We performed DFT-based Migdal-Eliashberg calculations of $T_{c}$ for bulk $\mathrm{RuO}_{2}$ and commensurately strained $\mathrm{RuO}_{2}(110)$ that indeed indicate epitaxial strain can enhance $T_{c}$ by several orders of magnitude. For bulk $\mathrm{RuO}_{2}$, we find that the empirical Coulomb pseudopotential must satisfy $\mu^{*}>0.30$ to be compatible with the experimentally measured least upper bound on $T_{c}\left(T_{c}<0.3 \mathrm{~K}^{15}\right)$. For this range of $\mu^{*}, T_{c}$ for $\mathrm{RuO}_{2}(110)$ can be as high as $7 \mathrm{~K}$ (Supplementary Note 9 and Supplementary Fig. 16). A robust strain-induced enhancement of the electron-phonon coupling $\lambda_{\mathrm{el}-\mathrm{ph}}$ boosts $T_{c}$ by a factor of 20 (for $\mu^{*}=0.30$ ), and this ratio becomes even larger for higher values of $\mu^{*}$-e.g., for $\mu^{*}=0.37$, $T_{c}(110) / T_{c}$ (bulk) $\left.=5 \mathrm{~K} / 5 \mathrm{mK}\right)$. Although these estimations of $T_{c}$ are broadly consistent with our experimental findings, conventional superconductivity in $\mathrm{RuO}_{2}$ remains a working hypothesis until measurements of the order parameter are possible.

In principle, assuming that all Fermi liquids are eventually unstable towards some channel(s) of Cooper pairing at sufficiently low temperatures and magnetic fields (including internal fields arising from magnetic impurities), the strain-stabilized superconductivity observed here in $\mathrm{RuO}_{2}$ is not strictly a change in the ground state of the system. For our purposes, however, extremely low temperatures and fields below what are experimentally achievable can be regarded as effectively zero, justifying our use of phrases such as strain-induced superconductivity interchangeably with huge enhancement of critical temperature. If we limit the scope of this semantic discussion to conventional, 


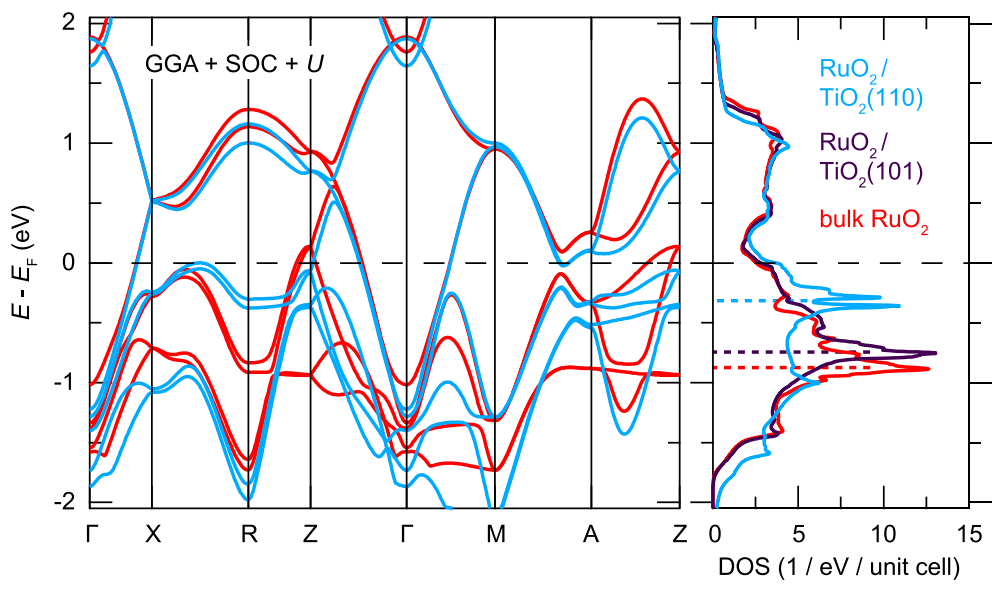

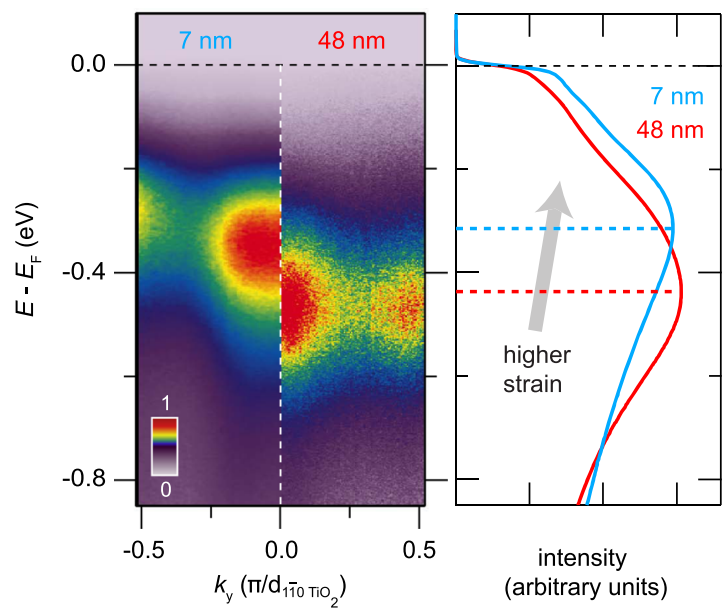

Fig. 4 Strain-induced changes to the electronic structure of $\mathbf{R u O}_{\mathbf{2}}$. a DFT $+U(U=2 \mathrm{eV}$ ) band structures and corresponding density of states (DOS) traces for bulk $\mathrm{RuO}_{2}$ and epitaxially strained $\mathrm{RuO}_{2}(110)$ and $\mathrm{RuO}_{2}(101)$ thin films. The $\mathrm{RuO}_{2}(101)$ results are omitted from the spaghetti plot for clarity since they are very similar to bulk. $\mathbf{b}$ Comparison of $E(k)$ spectra along the cut shown in Fig. 3e for two different $\mathrm{RuO}_{2}(110)$ samples: a highly strained $7 \mathrm{~nm}$ thick film (left), and a partially strain-relaxed $48 \mathrm{~nm}$ thick film (right). The false color scale used to visualize the intensities in each spectrum is defined and normalized in the same way as in Fig. 3. c As an approximate proxy of the total DOS, for these samples we plot the energy distribution curves of photoemission intensity averaged over the entire region of $\mathbf{k}$-space probed experimentally with $21.2 \mathrm{eV}$ photons (cf. Fig. 3c), which demonstrate that the epitaxial strains imposed by $\mathrm{TiO}_{2}(110)$ substrates shift $d_{\|}$states towards $E_{F}$ and thereby increase $N\left(E_{F}\right)$.

non-sign-changing ( $s$-wave) order parameters, we note that in the presence of Coulomb repulsion and other effects, an instability towards $s$-wave superconductivity is not present in every system; the electron-phonon coupling generally must exceed some finite critical value. In the present context, the effects of strain reported in this article might be boosting the electron-phonon coupling above the critical value appropriate for $\mathrm{RuO}_{2}$, thus inducing a new $s$-wave state that is absent (even in theory) for the unstrained material.

We believe our results demonstrate that a promising strategy to create new transition-metal superconductors is to apply judiciously chosen anisotropic strains that modulate degeneracies among $d$ orbitals near $E_{F}$. Many classic studies of conventional superconductors that have nearly-free-electron states spanning $E_{F}$ derived from $(s, p)$ orbitals actually show decreases in $T_{c}$ under hydrostatic pressure ${ }^{39}$, due to lattice stiffening dominating over any pressure-induced changes to the Hopfield parameter ${ }^{40}$. In a limited number of elemental metals where $T_{c}$ monotonically increases under pressure (such as vanadium ${ }^{41}$ ), pressure-induced electron transfer between $s \rightarrow d$ orbitals has been suggested as a likely cause of the enhanced transition temperatures ${ }^{3}$; a drawback of this approach, however, is that large pressures of $\gtrsim 10 \mathrm{GPa}$ are typically required to, e.g., double $T_{c}$. More recently, measurements on single crystals of the unconventional superconductor $\mathrm{Sr}_{2} \mathrm{RuO}_{4}$ have shown that appropriately oriented uniaxial pressures of only $\approx 1 \mathrm{GPa}$ can boost $T_{c}$ by more than a factor of two ${ }^{42}$. Independent of the underlying mechanism, it appears that anisotropic strains may prove to be significantly more efficacious than hydrostatic pressure for tuning superconductivity in multi-orbital systems, as shown here for $\mathrm{RuO}_{2}$, as well as in $\mathrm{Sr}_{2} \mathrm{RuO}_{4}$.

Sizable coupling between the lattice and electronic degrees of freedom in rutile-like crystal structures has been well established both theoretically ${ }^{26}$ and experimentally in $\mathrm{VO}_{2}$, where straininduced variations in the orbital occupancies can be used to modify the metal-insulator transition temperature by $\delta T_{\mathrm{MIT}} \approx 70$ $\mathrm{K}^{43,44}$. Therefore, it may be promising to explore other less strongly correlated (i.e., $4 d$ and $5 d$ ) rutile compounds such as $\mathrm{MoO}_{2}$ for strain-stabilized superconductivity, instead of employing chemical doping ${ }^{45-47}$. Finally, since $\mathrm{RuO}_{2} / \mathrm{TiO}_{2}(110)$ is the first known stoichiometric superconductor within the rutile family, further optimization of the superconductivity may enable the creation of structures that integrate superconductivity with other functional properties that have been extensively studied in other rutile compounds, such as high photocatalytic efficiency, half-metallic ferromagnetism, and large spin Hall conductivities.

\section{Methods}

Film synthesis. Epitaxial thin films of $\mathrm{RuO}_{2}$ were synthesized on various orientations of rutile $\mathrm{TiO}_{2}$ substrates using a GEN10 reactive oxide MBE system (Veeco Instruments). Prior to growth, $\mathrm{TiO}_{2}$ substrates (Crystec, $\mathrm{GmbH}$ ) were cleaned with organic solvents, etched in acid, and annealed in air to produce starting surfaces with step-terrace morphology, following the methods in Ref. ${ }^{48}$. Elemental ruthenium (99.99\% purity, ESPI Metals) was evaporated using an electron-beam evaporator in background oxidant partial pressures of $1 \times 10^{-6}-5 \times 10^{-6}$ Torr of distilled ozone $\left(\approx 80 \% \mathrm{O}_{3}+20 \% \mathrm{O}_{2}\right)$ at substrate temperatures of $250-400{ }^{\circ} \mathrm{C}$, as measured by a thermocouple. Reflection high-energy electron diffraction was used to monitor the surface crystallinity of the films in situ and showed characteristic oscillations in intensity during most of the $\mathrm{Ru}$ deposition, indicating a layer-bylayer growth mode following the initial nucleation of several-monolayer-thick $\mathrm{RuO}_{2}$ islands ${ }^{49}$.

Film characterization. The crystal structures of all $\mathrm{RuO}_{2}$ thin-film samples were characterized via lab-based $\mathrm{x}$-ray diffraction (XRD) measurements with $\mathrm{Cu}-\mathrm{K} \alpha$ radiation (Rigaku SmartLab and Malvern Panalytical Empyrean diffractometers). Four-point-probe electrical transport measurements were conducted from $300 \mathrm{~K}$ down to a base temperature of $0.4 \mathrm{~K}$ using a Physical Properties Measurement System equipped with a He-3 refrigerator (Quantum Design). All $\mathrm{RuO}_{2} / \mathrm{TiO}_{2}(110)$ samples were superconducting with $T_{c}$ s ranging from 0.5 to $2.4 \mathrm{~K}$, except for ultrathin films with residual resistivities $\rho_{0} \gtrsim 40 \mu \Omega-\mathrm{cm}$, as shown in Fig. 2 and Supplementary Fig. 9.

A subset of films studied by XRD and transport were also characterized in situ by ARPES and low-energy electron diffraction (LEED). For these measurements, films were transferred under ultrahigh vacuum immediately following growth to an analysis chamber with a base pressure of $5 \times 10^{-11}$ Torr equipped with a helium plasma discharge lamp, a hemispherical electron analyzer (VG Scienta R4000), and a four-grid LEED optics (SPECS ErLEED 150).

A subset of films studied by XRD and transport were also imaged using crosssectional STEM. Cross-sectional specimens were prepared using the standard focused ion beam (FIB) lift-out process on a Thermo Scientific Helios G4 X FIB. High-angle annular dark-field STEM (HAADF-STEM) images were acquired on an aberration-corrected FEI Titan Themis at $300 \mathrm{keV}$ with a probe convergence semiangle of $21.4 \mathrm{mrad}$ and inner and outer collection angles of 68 and $340 \mathrm{mrad}$.

Electronic structure calculations. Non-magnetic DFT calculations for the electronic structure of $\mathrm{RuO}_{2}$ were performed using the Quantum ESPRESSO software 
package ${ }^{50,51}$ with fully relativistic ultrasoft pseudopotentials for $\mathrm{Ru}$ and $\mathrm{O}^{52}$. We represented the Kohn-Sham wavefunctions in a basis set of plane waves extending up to a kinetic energy cutoff of $60 \mathrm{Ry}$, and used a cutoff of $400 \mathrm{Ry}$ for representing the charge density. Brillouin zone integrations were carried out on an $8 \times 8 \times 12 k$ mesh with $70 \mathrm{meV}$ of Gaussian smearing. Perdew, Burke, and Ernzerhof s parametrization of the generalized gradient approximation was employed as the exchange-correlation functional ${ }^{53}$, supplemented by an on-site correction of $+U_{\text {eff }}=U-J=2 \mathrm{eV}$ within spheres surrounding the Ru sites, following Ref. ${ }^{13}$.

After obtaining self-consistent Kohn-Sham eigenstates via DFT, we used the pw2wannier and Wannier90 $\operatorname{codes}^{54}$ to construct 20 Wannier functions spanning the manifold of eigenstates surrounding $E_{F}(20=10 d$-orbitals per Ru atom $\times 2$ $\mathrm{Ru}$ atoms per unit cell). Following Ref. ${ }^{55}$, to account for the non-symmorphic space group symmetries of rutile crystal structures, we referenced the trial orbitals employed in the Wannierisation routine to locally rotated coordinate systems centered on the two Ru sites within each unit cell. Orbital designations employed in the main text such as $d_{\|}$and $\left(d_{\mathrm{xz}}, d_{\mathrm{yz}}\right)$ refer to projections onto this basis of Wannier functions. The more computationally efficient Wannier basis was used to calculate quantities that required dense $k$ meshes to be properly converged, such as the projected Fermi surface in Fig. $3 \mathrm{~d}(51 \times 51 \times 51 k$-mesh $)$ and the near- $E_{F}$ density of states traces in Fig. $4 \mathrm{a}(32 \times 32 \times 48 k$-meshes $)$.

Because the $\mathrm{RuO}_{2}$ samples studied in this work are thin films subject to biaxial epitaxial strains imposed by differently oriented rutile $\mathrm{TiO}_{2}$ substrates, we performed DFT + Wannier calculations of the electronic structure for several different crystal structures of $\mathrm{RuO}_{2}$ as described in Supplementary Note 5 and Supplementary Table 1 . We used the ISOTROPY software package ${ }^{56}$ to study distortions of the parent tetragonal rutile crystal structure that are induced in biaxially strained thin films. Crystal structures and Wannier functions were visualized using the VESTA software package ${ }^{57}$.

Electron-phonon coupling calculations. To generate the inputs required for the electron-phonon coupling calculations described below, first-principles electronic structure and phonon calculations were performed using the Quantum ESPRESSO software package with norm-conserving pseudopotentials and plane-wave basis sets $^{50,51}$. Here we employed a kinetic energy cutoff of $160 \mathrm{Ry}$, an electronic momentum $k$-point mesh of $16 \times 16 \times 24,20 \mathrm{meV}$ of Methfessel-Paxton smearing for the occupation of the electronic states, and a tolerance of $10^{-10} \mathrm{eV}$ for the total energy convergence. The generalized gradient approximation as implemented in the PBEsol functional ${ }^{58}$ was employed as the exchange-correlation functional. For the Wannier interpolation, we used an interpolating electron-momentum mesh of $8 \times 8 \times 12$ and a phonon-momentum mesh of $2 \times 2 \times 3$. Results for bulk $\mathrm{RuO}_{2}$ were calculated using the crystal structure that minimizes the DFT-computed total energy with the PBEsol functional: $(a=4.464 \AA, c=3.093 \AA)$ and $x_{\text {oxygen }}=0.3062$. Results for strained $\mathrm{RuO}_{2}(110)$ were calculated by changing the lattice constants of this simulated bulk crystal structure by $+2.3 \%$ along $[1 \overline{1} 0],-4.7 \%$ along [001], $+2.2 \%$ along [110], and setting $x_{\text {oxygen }}=y_{\text {oxygen }}=0.2996$. The lattice parameter along [110] and internal coordinates of this simulated $\mathrm{RuO}_{2}(110)$ structure were determined by allowing the structure to relax so as to (locally) minimize the DFTcomputed total energy.

Electron-phonon coupling calculations were performed using the EPW $\operatorname{code}^{59}$, using an interpolated electron-momentum mesh of $32 \times 32 \times 48$ and an interpolated phonon-momentum mesh of $8 \times 8 \times 12$. The isotropic Eliashberg spectral function $\alpha^{2} F(\omega)$ and total electron-phonon coupling constant $\lambda_{\mathrm{el}-\mathrm{ph}}$ (integrated over all phonon modes and wavevectors) were calculated with a phonon smearing of $0.2 \mathrm{meV}$. From the calculated $\alpha^{2} F(\omega)$ and $\lambda_{\mathrm{el}-\mathrm{ph}}$, we estimated the superconducting transition temperature using the semi-empirical McMillanAllen-Dynes formula ${ }^{60,61}$ :

$$
T_{c}=\frac{\omega_{\log }}{1.2} \exp \left[-\frac{1.04\left(1+\lambda_{\mathrm{el}-\mathrm{ph}}\right)}{\lambda_{\mathrm{el}-\mathrm{ph}}-\mu^{*}\left(1+0.62 \lambda_{\mathrm{el}-\mathrm{ph}}\right)}\right]
$$

\section{Data availability}

The data supporting the findings of this study are available within the paper and supplementary information. Data connected to the study from PARADIM facilities are available at paradim.org. Any additional data connected to the study are available from the corresponding author upon reasonable request.

Received: 19 August 2020; Accepted: 19 November 2020; Published online: 04 January 2021

\section{References}

1. Carbotte, J. P. Properties of boson-exchange superconductors. Rev. Mod. Phys. 62, 1027-1157 (1990).

2. Morel, P. \& Anderson, P. W. Calculation of the superconducting state parameters with retarded electron-phonon interaction. Phys. Rev. 125, 1263-1271 (1962).
3. Hamlin, J. J. Superconductivity in the metallic elements at high pressures. Phys. C 514, 59-76 (2015).

4. Burganov, B. et al. Strain control of fermiology and many-body interactions in two-dimensional ruthenates. Phys. Rev. Lett. 116, 197003 (2016).

5. Ohtomo, A. \& Hwang, H. Y. A high-mobility electron gas at the $\mathrm{LaAlO}_{3} /$ $\mathrm{SrTiO}_{3}$ heterointerface. Nature 427, 423 (2004).

6. Kawasaki, J. K. et al. Rutile $\mathrm{IrO}_{2} / \mathrm{TiO}_{2}$ superlattices: a hyperconnected analog to the Ruddelsden-Popper structure. Phys. Rev. Mater. 2, 054206 (2018).

7. Lock, J. M. \& Bragg, W. L. Penetration of magnetic fields into superconductors III. Measurements on thin films of tin, lead and indium. Proc. R. Soc. Lond. Ser. A. 208, 391-408 (1951).

8. Locquet, J.-P. et al. Doubling the critical temperature of $\mathrm{La}_{1.9} \mathrm{Sr}_{0.1} \mathrm{CuO}_{4}$ using epitaxial strain. Nature 394, 453 (1998).

9. $\mathrm{Si}, \mathrm{W} ., \mathrm{Li}, \mathrm{H} .-\mathrm{C}$. \& Xi, X. X. Strain and oxygenation effects on superconductivity of $\mathrm{La}_{1.85} \mathrm{Sr}_{0.15} \mathrm{CuO}_{4}$ thin films. Appl. Phys. Lett. 74, 2839-2841 (1999).

10. Si, W. \& Xi, X. X. Epitaxial-strain-induced insulator-superconductor transition in undoped and lightly doped $\mathrm{La}_{2} \mathrm{CuO}_{4}$. Appl. Phys. Lett. 78, 240-242 (2001).

11. Bozovic, I., Logvenov, G., Belca, I., Narimbetov, B. \& Sveklo, I. Epitaxial strain and superconductivity in $\mathrm{La}_{2-x} \mathrm{Sr}_{x} \mathrm{CuO}_{4}$ thin films. Phys. Rev. Lett. 89, 107001 (2002).

12. Engelmann, J. et al. Strain induced superconductivity in the parent compound $\mathrm{BaFe}_{2} \mathrm{As}_{2}$. Nat. Commun. 4, 2877 (2013).

13. Berlijn, T. et al. Itinerant antiferromagnetism in $\mathrm{RuO}_{2}$. Phys. Rev. Lett. 118, 077201 (2017).

14. Burdett, J. K., Hughbanks, T., Miller, G. J., Richardson, J. W. \& Smith, J. V. Structural-electronic relationships in inorganic solids: powder neutron diffraction studies of the rutile and anatase polymorphs of titanium dioxide at 15 and 295 K. J. Am. Chem. Soc. 109, 3639-3646 (1987).

15. Lin, J. J. et al. Low temperature electrical transport properties of $\mathrm{RuO}_{2}$ and $\mathrm{IrO}_{2}$ single crystals. J. Phys.: Condens. Matter 16, 8035 (2004).

16. Ryden, W. D., Lawson, A. W. \& Sartain, C. C. Electrical transport properties of $\mathrm{IrO}_{2}$ and $\mathrm{RuO}_{2}$. Phys. Rev. B 1, 1494-1500 (1970).

17. Pinto, N. et al. Dimensional crossover and incipient quantum size effects in superconducting niobium nanofilms. Sci. Rep. 8, 4710 (2018).

18. Meyer, T. L., Jiang, L., Park, S., Egami, T. \& Lee, H. N. Strain-relaxation and critical thickness of epitaxial $\mathrm{La}_{1.85} \mathrm{Sr}_{0.15} \mathrm{CuO}_{4}$ films. APL Mater. 3, 126102 (2015).

19. Gozar, A. et al. High-temperature interface superconductivity between metallic and insulating copper oxides. Nature 455, 782-785 (2008).

20. He, S. et al. Phase diagram and electronic indication of high-temperature superconductivity at $65 \mathrm{~K}$ in single-layer FeSe films. Nat. Mater. 12, 605-610 (2013).

21. Lee, J. J. et al. Interfacial mode coupling as the origin of the enhancement of $T_{c}$ in FeSe films on $\mathrm{SrTiO}_{3}$. Nature 515, 245-248 (2014).

22. Paik, $\mathrm{H}$. et al. Transport properties of ultra-thin $\mathrm{VO}_{2}$ films on (001) $\mathrm{TiO}_{2}$ grown by reactive molecular-beam epitaxy. Appl. Phys. Lett. 107, 163101 (2015).

23. Quackenbush, N. F. et al. Reducing orbital occupancy in $\mathrm{VO}_{2}$ suppresses Mott physics while Peierls distortions persist. Phys. Rev. B 96, 081103 (2017).

24. Yoshimatsu, K., Sakata, O. \& Ohtomo, A. Superconductivity in $\mathrm{Ti}_{4} \mathrm{O}_{7}$ and $\gamma-$ $\mathrm{Ti}_{3} \mathrm{O}_{5}$ films. Sci. Rep. 7, 12544 (2017).

25. Goodenough, J. B. The two components of the crystallographic transition in $\mathrm{VO}_{2}$. J. Solid State Chem. 3, 490-500 (1971).

26. Eyert, V., Horny, R., Hoeck, K.-H. \& Horn, S. Embedded Peierls instability and the electronic structure of $\mathrm{MoO}_{2}$. J. Phys.: Condens. Matter 12, 4923 (2000).

27. Mackenzie, A. P. et al. Quantum oscillations in the layered perovskite superconductor $\mathrm{Sr}_{2} \mathrm{RuO}_{4}$. Phys. Rev. Lett. 76, 3786-3789 (1996).

28. Mravlje, J. et al. Coherence-incoherence crossover and the massrenormalization puzzles in $\mathrm{Sr}_{2} \mathrm{RuO}_{4}$. Phys. Rev. Lett. 106, 096401 (2011).

29. Tamai, A. et al. High-resolution photoemission on $\mathrm{Sr}_{2} \mathrm{RuO}_{4}$ reveals correlation-enhanced effective spin-orbit coupling and dominantly local selfenergies. Phys. Rev. X 9, 021048 (2019).

30. Ricco, S. et al. In situ strain tuning of the metal-insulator-transition of $\mathrm{Ca}_{2} \mathrm{RuO}_{4}$ in angle-resolved photoemission experiments. Nat. Commun. 9, 4535 (2018).

31. Sutter, D. et al. Orbitally selective breakdown of Fermi liquid quasiparticles in $\mathrm{Ca}_{1.8} \mathrm{Sr}_{02} \mathrm{RuO}_{4}$. Phys. Rev. B 99, 121115 (2019).

32. Sutter, D. et al. Hallmarks of Hunds coupling in the Mott insulator $\mathrm{Ca}_{2} \mathrm{RuO}_{4}$. Nat. Commun. 8, 15176 (2017)

33. Jovic, V. et al. Dirac nodal lines and flat-band surface state in the functional oxide $\mathrm{RuO}_{2}$. Phys. Rev. B 98, 241101 (2018)

34. Passenheim, B. C. \& McCollum, D. C. Heat capacity of $\mathrm{RuO}_{2}$ and $\mathrm{IrO}_{2}$ between 0.54 and 10 K. J. Chem. Phys. 51, 320-321 (1969).

35. Glassford, K. M. \& Chelikowsky, J. R. Electron transport properties in $\mathrm{RuO}_{2}$ rutile. Phys. Rev. B 49, 7107-7114 (1994). 
36. Marcus, S. M. \& Butler, S. R. Measurement of the de Haas-van Alphen effect in the rutile structure $\mathrm{RuO}_{2}$. Phys. Lett. A 26, 518-519 (1968).

37. Slivka, R. T. \& Langenberg, D. N. Azbel'-Kaner cyclotron resonance in ruthenium dioxide. Phys. Lett. A 28, 169-170 (1968).

38. Graebner, J. E., Greiner, E. S. \& Ryden, W. D. Magnetothermal oscillations in $\mathrm{RuO}_{2}, \mathrm{OsO}_{2}$, and $\mathrm{IrO}_{2}$. Phys. Rev. B 13, 2426-2432 (1976).

39. Smith, T. F. \& Chu, C. W. Will pressure destroy superconductivity? Phys. Rev. 159, 353-358 (1967).

40. Hopfield, J. J. Angular momentum and transition-metal superconductivity. Phys. Rev. 186, 443-451 (1969).

41. Ishizuka, M., Iketani, M. \& Endo, S. Pressure effect on superconductivity of vanadium at megabar pressures. Phys. Rev. B 61, R3823-R3825 (2000).

42. Steppke, A. et al. Strong peak in $T_{c}$ of $\mathrm{Sr}_{2} \mathrm{RuO}_{4}$ under uniaxial pressure. Science 355, eaaf9398 (2017)

43. Muraoka, Y. \& Hiroi, Z. Metal-insulator transition of $\mathrm{VO}_{2}$ thin films grown on $\mathrm{TiO}_{2}$ (001) and (110) substrates. Appl. Phys. Lett. 80, 583-585 (2002).

44. Aetukuri, N. B. et al. Control of the metal-insulator transition in vanadium dioxide by modifying orbital occupancy. Nat. Phys. 9, 661-666 (2013).

45. Alves, L. M. S. et al. Unconventional metallic behavior and superconductivity in the K-Mo-O system. Phys. Rev. B 81, 174532 (2010).

46. Alves, L. M. S. et al. Superconductivity and magnetism in the $\mathrm{K}_{x} \mathrm{MoO}_{2-\delta}$. J. Appl. Phys. 112, 073923 (2012).

47. Parker, D., Idrobo, J. C., Cantoni, C. \& Sefat, A. S. Evidence for superconductivity at $T_{c}=12 \mathrm{~K}$ in oxygen-deficient $\mathrm{MoO}_{2-\delta}$ and properties of molybdenum arsenide and oxide binaries. Phys. Rev. B 90, 054505 (2014).

48. Yamamoto, Y., Nakajima, K., Ohsawa, T., Matsumoto, Y. \& Koinuma, H Preparation of atomically smooth $\mathrm{TiO}_{2}$ single crystal surfaces and their photochemical property. Jpn. J. Appl. Phys. 44, L511 (2005).

49. He, Y., Langsdorf, D., Li, L. \& Over, H. Versatile model system for studying processes ranging from heterogeneous to photocatalysis: epitaxial $\mathrm{RuO}_{2}(110)$ on $\mathrm{TiO}_{2}(110)$. J. Phys. Chem. C 119, 2692-2702 (2015).

50. Giannozzi, P. et al. QUANTUM ESPRESSO: a modular and open-source software project for quantum simulations of materials. J. Phys.: Condens. Matter 21, 395502 (2009).

51. Giannozzi, P. et al. Advanced capabilities for materials modelling with Quantum ESPRESSO. J. Phys.: Condens. Matter 29, 465901 (2017).

52. Dal Corso, A. Pseudopotentials periodic table: from $\mathrm{H}$ to Pu. Comp. Mater. Sci. 95, 337-350 (2014).

53. Perdew, J. P., Burke, K. \& Ernzerhof, M. Generalized gradient aproximation made simple. Phys. Rev. Lett. 77, 3865-3868 (1996).

54. Mostofi, A. A. et al. An updated version of wannier90: a tool for obtaining maximally-localised Wannier functions. Comput. Phys. Commun. 185, 2309-2310 (2014).

55. Eyert, $\mathrm{V}$. The metal-insulator transitions of $\mathrm{VO}_{2}:$ a band theoretical approach. Ann. der Phys. 11, 650-704 (2002).

56. Stokes, H. T., Hatch, D. M. \& Campbell, B. J. ISOTROPY Software Suite. iso. byu.edu.

57. Momma, K. \& Izumi, F. VESTA 3 for three-dimensional visualization of crystal, volumetric and morphology data. J. Appl. Crystallogr. 44, 1272-1276 (2011).

58. Perdew, J. P. et al. Restoring the density-gradient expansion for exchange in solids and surfaces. Phys. Rev. Lett. 100, 136406 (2008).

59. Ponce, S., Margine, E. R., Verdi, C. \& Giustino, F. EPW: electron-phonon coupling, transport and superconducting properties using maximally localized Wannier functions. Comput. Phys. Commun. 209, 116-133 (2016).

60. McMillan, W. L. Transition temperature of strong-coupled superconductors. Phys. Rev. 167, 331-344 (1968).

61. Allen, P. B. \& Dynes, R. C. Transition temperature of strong-coupled superconductors reanalyzed. Phys. Rev. B 12, 905-922 (1975).

\section{Acknowledgements}

The authors thank Y. Li for assistance with electrical transport measurements. This work was supported through the National Science Foundation (Platform for the Accelerated Realization, Analysis, and Discovery of Interface Materials, PARADIM) under Cooperative Agreement No. DMR-1539918, NSF DMR-1709255, the Air Force Office of Scientific Research Grant No. FA9550-15-1-0474, and the Department of Energy (Award No. DE-SC0019414). This research was funded in part by the Gordon and Betty Moore Foundation's EPiQS Initiative through Grant Nos. GBMF3850 and GBMF9073 to Cornell University. This work made use of the Cornell Center for Materials Research (CCMR) Shared Facilities, which are supported through the NSF MRSEC Program (No. DMR-1719875). The FEI Titan Themis 300 was acquired through NSF-MRI-1429155, with additional support from Cornell University, the Weill Institute, and the Kavli Institute at Cornell. Device fabrication and substrate preparation were performed in part at the Cornell NanoScale Facility, a member of the National Nanotechnology Coordinated Infrastructure (NNCI), which is supported by the NSF (Grant No. ECCS-1542081).

\section{Author contributions}

H.P., N.J.S., and H.P.N. synthesized the samples by MBE. J.P.R., H.P., N.J.S., and H.P.N characterized the samples by XRD. J.P.R., L.M., and Y.L. characterized the samples by electrical transport; L.M. lithographically patterned resistivity bridges on select films. J.P.R., J.K.K., J.N.N., and B.D.F. characterized the samples by ARPES and LEED. B.H.G. characterized the samples by STEM, J.P.R. performed DFT calculations of the electronic structure, and B.P. performed DFT-based calculations of the electron-phonon coupling. L.F.K., D.G.S., and K.M.S. supervised the various aspects of this project. J.P.R. and K.M.S. wrote the manuscript with input from all authors.

\section{Competing interests}

The authors declare no competing interests.

\section{Additional information}

Supplementary information is available for this paper at https://doi.org/10.1038/s41467020-20252-7.

Correspondence and requests for materials should be addressed to J.P.R. or K.M.S.

Peer review information Nature Communications thanks the anonymous reviewers for their contribution to the peer review of this work.

Reprints and permission information is available at http://www.nature.com/reprints

Publisher's note Springer Nature remains neutral with regard to jurisdictional claims in published maps and institutional affiliations.

pen Access This article is licensed under a Creative Commons Attribution 4.0 International License, which permits use, sharing, adaptation, distribution and reproduction in any medium or format, as long as you give appropriate credit to the original author(s) and the source, provide a link to the Creative Commons license, and indicate if changes were made. The images or other third party material in this article are included in the article's Creative Commons license, unless indicated otherwise in a credit line to the material. If material is not included in the article's Creative Commons license and your intended use is not permitted by statutory regulation or exceeds the permitted use, you will need to obtain permission directly from the copyright holder. To view a copy of this license, visit http://creativecommons.org/ licenses/by/4.0/.

(C) The Author(s) 2021 